\title{
Using Gavish-Grave LP to Formulate the Directed Black and White Traveling Salesman Problem
}

\author{
He Jiang ${ }^{1}$, XianChao Zhang ${ }^{1}$, MingChu Li ${ }^{1}$, and HaoYang Che ${ }^{2}$ \\ ${ }^{1}$ School of Software, Dalian University of Technology, Dalian 116621, China \\ \{xczhang, jianghe, mingchul\}@dlut.edu.cn \\ ${ }^{2}$ Institute of Software, The Chinese Academy of Sciences, Beijing 100080, China \\ grandiose11@msn.com
}

\begin{abstract}
The black and white traveling salesman problem (BWTSP) is a new class of NP-hard problem arising from work on airline scheduling and telecommunication fiber networks. The existing Ghiani LP for the undirected BWTSP contains an exponential number of constraints. For a special case of the directed BWTSP whose $L=+\infty$, the LP with polynomial number of constraints could be obtained by transforming it to an asymmetric traveling salesman problem with replenishment arcs (RATSP), whereas there exists no LP for the directed BWTSP in its general form. This paper proposes a LP with $3 n^{2}+2 n$ constraints only for the directed BWTSP in such a way that, by reducing the problem to an asymmetric traveling salesman problem (ATSP), we add $n^{2}$ cardinality constraints and $n^{2}$ length constraints to the existing GavishGrave LP for the ATSP. The new LP is also valid for the undirected BWTSP when viewed as a special case of the directed BWTSP.
\end{abstract}

Keywords: Black and white traveling salesman problem, Linear programming, Gavish-Grave LP.

\section{Introduction}

The black and white traveling salesman problem (BWTSP) is a generalization of the well known traveling salesman problem (TSP) and is therefore NP-hard. Given a directed graph $G=(V, A)$ or an undirected graph $G=(V, E)$ with vertex set $V=\{1, \cdots, n\}$, arc set $A=\{(i, j): i, j \in V, i \neq j\}$ or edge set $E=\{(i, j): i, j \in V, i \neq j\}$, and costs on the $\operatorname{arcs} c \in R^{|A|}$ or costs on the edges $c \in R^{|E|}$, the BWTSP is to find a shortest Hamiltonian tour on $G$. However the tour must satisfy additional constraints i) cardinality constraints: the vertex set $V$ is partitioned into black vertices, denoted by $B(|B| \geq 2)$ and white vertices, denoted by $W=V \backslash B$, the number of white vertices between two consecutive black vertices on the tour does not exceed a positive integer $Q$, and ii) length constraints: the length of any path or chain between two consecutive black vertices does not exceed a positive value $L$.

An application of the BWTSP arises in the design of telecommunication fiber networks using the SONET technology $[1,2]$. When designing survivable fiber 
networks using the SONET technology, one seeks a shortest Hamiltonian cycle comprising hubs (white vertices) and ring offices (black vertices) with upper bounds on both the number of hubs and chain length between consecutive ring offices. Aside the above-mentioned application, the scheduling of airline operations that incorporate maintenance connections [3] can also be modeled as a BWTSP.

Ghiani et al. have shown a linear programming (LP) with exponential constraints for the undirected BWTSP, whereas no LP exists for the directed BWTSP in its general form. With the special case of the directed BWTSP whose $Q=L=+\infty$ is associated extensive existing LPs by reverting to the usual ATSP (see [5]). Dantzig et al. [6] introduced a conventional LP with $2^{n-1}-n-1$ constraints in 1954. Miller et al. [7] presented a sequential LP with $n^{2}-n+2$ constraints in 1960. A single commodity flow based LP with $n(n+2)$ constraints was proposed by Gavish et al. [8] in 1978. In 1983 Finke et al. [9] developed a two-commodity flow based LP with $n(n+4)$ constraints. Wong [10] and Claus [11] described the multi-commodity flow based LP with $n^{3}+n^{2}+6 n-3$ constraints, respectively, in 1980 and 1984. Besides a time staged LP with $2 n^{2}-n+3$ constraints by Vajda [12] in 1961, Fox et al. [13] showed two time staged LPs in 1980, one with $4 n-1$ constraints, the other with $n(n+2)$ constraints.

When $L=+\infty$, the directed BWTSP reduced to an asymmetric traveling salesman problem with replenishment arcs (RATSP). The natural arc LP for the RATSP was developed by Zhu [14] based on the DFJ formulation for the ATSP in 1994. Boland et al. [15] proposed a path LP for the same problem in 2000. Both of the previously mentioned LPs involve exponentially many variables and constraints. Vicky [16] strengthened the LP by Zhu, and proposed a polynomial-size LP.

In this paper we propose a new LP with polynomial constraints for the directed BWTSP in its general form. The directed BWTSP reduces to an ATSP when $Q=L=\infty$, thus we can derive our LP from the Gavish-Grave LP [8] for the ATSP.

The new $n^{2}$ formulations of cardinality constraints are obtained in such a way that, by introducing cardinality flow representing the number of white vertices quota remaining, we investigate the conditions that the arcs on the tour should be subject to. Similarly, we obtain $n^{2}$ formulations of length constraints. Therefore, we establish the new LP with $3 n^{2}+2 n$ constraints for the original problem. The new LP is also valid for the undirected BWTSP when viewed as a special case of the directed BWTSP. The results of the paper can promote the complete algorithm design of the BWTSP.

The remainder of this paper is organized as follows. In section 2, we describe the Gavish-Grave LP for the ATSP. Cardinality constraints and length constraints are presented in section 3 and in section 4, respectively, followed by conclusions in section 5 .

\section{Gavish-Grave LP for the ATSP}

As mentioned before, the directed BWTSP reduces to an ATSP when $Q=L=\infty$, thus any solution to the directed BWTSP is feasible for the ATSP, and all inequalities 
for the ATSP are also valid for the directed BWTSP. Therefore, we are able to derive our LP from the Gavish-Grave LP.

We define $x_{i j}$ as a binary variable equal to 1 iff arc $(i, j)$ belongs to the solution. To eliminate subtours, we introduce $y_{i j}$ as a continuous variable which represents the commodity flow in arc $(i, j)$. The Gavish-Grave LP for the ATSP can be described as follows.

$$
\begin{array}{ll}
\min \sum_{i \neq j} c_{i j} x_{i j} & \forall j \in B \bigcup W \\
\text { s.t. } \sum_{i \neq j} x_{i j}=1 & \forall i \in B \bigcup W \\
\sum_{j \neq i} x_{i j}=1 & (i, j) \in A \\
x_{i j}=0 \text { or } 1 & \forall i, j \in B \bigcup W, i \neq j \\
0 \leq y_{i j} \leq(n-1) x_{i j} & \\
\sum_{j \neq 1} y_{1 j}=n-1 & \\
\sum_{i \neq j} y_{i j}-\sum_{z \neq j} y_{j z}=1 & \forall j \in B \bigcup W-\{1\}
\end{array}
$$

In this LP, constraints (2)-(4) are degree constraints which insure that exactly one arc enters and leaves each vertex. Whereas constraints (5)-(7) are called arc constraints which guarantee that the solution contains exactly one loop. Assume that the solution contains more than one loop, all of them distinct. Consider a loop $t=\left(i_{0}, i_{1}, \ldots, i_{k}, i_{0}\right)$ which does not contain vertex 1 . From (5) and (7) it follows that $y_{i_{j-1} i_{j}}-y_{i_{j} i_{(j+1) \bmod (k+1)}}=1$ for all $j=1, \ldots, k$, therefore we have $y_{i_{0} i_{1}}=y_{i_{k} i_{0}}+k$, nevertheless from (7) it follows that $y_{i_{k} i_{0}}-y_{i_{0} i_{1}}=1$. No loops can exist that do not contain vertex 1 ; since vertex 1 is contained in exactly one loop, thus at most one loop is generated.

The Gavish-Grave LP consists of $n(n+2)$ constraints, $n(n-1) 0-1$ variables and $n(n-1)$ continuous variables.

\section{Cardinality Constraints}

We define $p=\left(i_{0}, i_{1}, \ldots, i_{k}\right)$ as a black-to-black path with $\left(i_{j-1}, i_{j}\right) \in A$ for all $j=1, \ldots, k, i_{j} \neq i_{l}$ for all distinct $j, l \in\{0, \ldots, k\}$, and $i_{0}, i_{k} \in B, i_{j} \in W$ for all $j=1, \ldots, k-1$. We also define $V(p)=\left\{i_{0}, i_{1}, \ldots, i_{k}\right\}$ as the vertices on $p$, $A(p)=\left\{\left(i_{0}, i_{1}\right),\left(i_{1}, i_{2}\right), \ldots,\left(i_{k-1}, i_{k}\right)\right\}$ as the arcs on $p$, and $f(p)=c_{i_{0} i_{1}}+c_{i_{1} i_{2}}+\ldots+c_{i_{k-1} i_{k}}$ as the length of $p$. A black-to-black path $p$ is said to be feasible if the number of white 
vertices on it does not exceed $Q$, i.e. $|V(p)|-2 \leq Q$, and the length of it does not exceed $L$, i.e. $f(p) \leq L$. Otherwise it is said to be infeasible. Obviously, a feasible solution of the BWTSP consists of alternating feasible black-to-black paths.

To eliminate cardinality violation, we introduce extra cardinality flow $q_{i j}$ for each $(i, j) \in A$, which represents the number of white vertices quota remaining after visiting arc $(i, j)$. Then our cardinality constraints can be derived as follows.

The total cardinality flow into a white vertex $j \in W$ will be 1 more than that out of $j$. Therefore, we have the following constraint:

$$
\sum_{i \neq j} q_{i j}-\sum_{z \neq j} q_{j z}=1 \quad \forall j \in W
$$

And the total cardinality flow out of each black vertex $j \in B$ should not exceed $Q$ in order to guarantee that, the number of white vertices on the black-to-black path starting with vertex $j$ does not exceed $Q$.

$$
\sum_{i \neq j} q_{j i} \leq Q \quad \forall j \in B
$$

Last of all, $q_{i j}$ is bounded according to:

$$
0 \leq q_{i j} \leq Q x_{i j} \quad \forall i, j \in B \cup W, i \neq j
$$

Constraints (8), (9) and (10) are formulations of cardinality constraints which impose the maximum number restriction of white vertices between consecutive black vertices. These formulations consist of $n^{2}$ constraints and $n(n-1)$ new continuous variables.

Proposition 1. Constraints (8), (9) and (10) are valid for the directed BWTSP.

Proof. Assume that a solution of the directed BWTSP subject to constraints (8), (9) and (10) violates the cardinality constraints. Thus, there must be a black-to-black path $p=\left(i_{0}, i_{1}, \ldots, i_{k}\right)$ on the solution subject to $k-2 \geq Q$. From (8) and (10) it follows that $q_{i, i_{r+1}}=q_{i_{r+1} i_{r+2}}+1$ for all $r=0, \ldots, k-2$. Therefore, we have $q_{i_{0} i_{1}}=q_{i_{k-1} i_{k}}+k-1>Q$, which contradicts with constraint (9). Thus, no solution subject to (8), (9) and (10) could exist that does not satisfy cardinality constraints.

To show that a feasible solution of the directed BWTSP satisfies (8), (9) and (10), assume that $p=\left(i_{0}, i_{1}, \ldots, i_{k}\right)$ is a feasible black-to-black path on the solution; (8), (9) and (10) hold by assigning $q_{i_{r} i_{r+1}}=k-1-r, q_{i_{r} z}=0$ for all $\left(i_{r}, z\right) \notin A(p)$, $r=0, \ldots, k-1$.

\section{Length Constraints}

To eliminate length violation, we introduce length flow $l_{i j}$ for each $(i, j) \in A$, which represents the length quota remaining after visiting arc $(i, j)$. Our length constraints are derived as follows. 
If arc $(i, j) \in A(i \in W)$ belongs to the solution, i.e. if $x_{i j}=1$, the total length flow out of vertex $i$ will be $c_{i j}$ less than that into it. To guarantee this is satisfied, we require that if $x_{i j}=1$, then $\sum_{z \neq i} l_{i z}=\sum_{s \neq i} l_{s i}-c_{i j}$. Therefore, we have the following constraint:

$$
\sum_{z \neq i} l_{i z}=\sum_{s \neq i} l_{s i}-\sum_{z \neq i} c_{i z} x_{i z} \quad \forall i \in W
$$

If arc $(i, j) \in A(i \in B)$ belongs to the solution, i.e. if $x_{i j}=1$, the total length flow out of vertex $i$ will be considered "refreshed" after visiting $i$ and $l_{i j}$ will be set to $L-c_{i j}$. Thus, we obtain the following constraint:

$$
\sum_{z \neq i} l_{i z}=L-\sum_{z \neq i} c_{i z} x_{i z} \quad \forall i \in B
$$

Last of all, $l_{i j}$ is bounded according to:

$$
0 \leq l_{i j} \leq x_{i j} L \quad \forall i, j \in B \cup W, i \neq j
$$

Constraints (11), (12) and (13) are formulations of length constraints which impose the maximum length restriction between consecutive black vertices. These formulations consist of $n^{2}$ constraints and $n(n-1)$ new continuous variables.

Proposition 2. Constraints (11), (12) and (13) are valid for the directed BWTSP.

Proof. Assume that a solution subject to constraints (11), (12) and (13) violates the length constraints. Thus, there must be a black-to-black path $p=\left(i_{0}, i_{1}, \ldots, i_{k}\right)$ on the solution subject to $\sum_{0 \leq r \leq k-1} c_{i_{r} i_{r+1}}>L$ and $x_{i_{r} i_{r+1}}=1$ for all $r=0, \cdots, k-1$. From (11) it follows that $l_{i_{r+1} i_{r+2}}=l_{i_{r} i_{r+1}}-c_{i_{r+1} i_{r+2}}$ for all $r=0, \ldots, k-2$. Therefore, we have $l_{i_{k-1} i_{k}}=l_{i_{0} i_{1}}-\sum_{1 \leq r \leq k-1} c_{i_{r} i_{r+1}}$. From (12) it follows that $l_{i_{0} i_{1}}=L-c_{i_{0} i_{1}}$; hence, we have $l_{i_{k-1} i_{k}}=L-\sum_{0 \leq r \leq k-1} c_{i_{r} i_{r+1}}<0$, which contradicts with constraint (13). Thus, no solution subject to (11), (12) and (13) can exist that does not satisfy length constraints.

We can show that a feasible solution of the directed BWTSP satisfies (11), (12) and (13) in a similar way as given in the proof of Proposition 1. Given a feasible black-to-black path $p=\left(i_{0}, i_{1}, \ldots, i_{k}\right)$ on the solution, (11), (12) and (13) hold by assigning $l_{i_{r} i_{r+1}}=L-\sum_{0 \leq s \leq r} c_{i_{s} i_{s+1}}, l_{i_{r} z}=0$ for all $\left(i_{r}, z\right) \notin A(p), r=0, \ldots, k-1$.

\section{Conclusion}

In this paper, we have introduced a new LP for the directed BWTSP. Compared to the Ghiani LP with exponential constraints for the undirected BWTSP, our LP involves $3 n^{2}+2 n$ constraints only. The new LP is derived from the Gavish-Grave LP by 
defining two classes of flows: one represents the number of white vertices quota remaining, the other represents the length quota remaining.

Acknowledgements. This work was supported by Natural Science Foundation of China (No.60673066, 60673046, 60503003), the Natural Science Foundation of LiaoNing Province (No.20051082), and the Gifted Young Foundation of Dalian University of Technology.

\section{References}

1. Wasem OJ. An algorithm for designing rings in survivable fiber networks. IEEE Transactions on Reliability (1991) 40:428-32

2. Cosares S, Deutsch DN, Saniee I, Wasem OJ. SONET toolkit: a decision support system for designing robust and cost-effective fiber-optic networks. Interfaces (1995) 25(1):20-40

3. Talluri KT. The four-day aircraft maintenance routing problem. Transportation Science (1998) 32:43-53

4. Mak V, Boland N. Heuristic approaches to the asymmetric traveling salesman problem with replenishment arcs. International Transactions in Operation Research (2000) 7:43147.

5. Orman AJ, Williams HP. A survey of different integer programming formulations of the travelling salesman problem. Working Paper No: LSEOR 04.67, The London School of Economics and Political Science (2004)

6. Dantzig GG, Fulkerson DR and Johnson SM. Solutions of a large scale traveling salesman problem. Operations. Research (1954) 2:393-410

7. Miller CE, Tucker AW and Zemlin RA. Integer programming formulation of traveling salesman problem. Journal of ACM (1960) 3:326-329

8. Gavish B and Graves SC. The traveling salesman problem and related problems. Working Paper OR-078-78, Operations Research Center, MIT, Cambridge, MA (1978)

9. Finke KR, Gavish B and Graves SC. A two-commodity network flow approach to the traveling salesman problem. Combinatorics, Graph Theory and Computing, Proc. 14th South Eastern Conf., Atlantic University, Florida (1983)

10. Wong RT. Integer programming formulations of the traveling salesman problem. Proc. IEEE Conf. On Circuits and Computers (1980) 149-152

11. Claus A. A new formulation for the traveling salesman problem. SIAM Journal of Algorithm and Discrete Mathematics (1984) 5:21-25

12. Vajda S. Mathematical Programming. Addison-Wesley, London (1961)

13. Fox KR, Gavish B and Graves SC. An n-constraint formulation of the (time-dependent) traveling salesman problem. Operations Research (1980) 28:1018-1021

14. Zhu, Z. The aircraft rotation problem. PhD thesis, Georgia Institute of Technology (1994)

15. Boland NL, Clarke LW, Nemhauser GL. The asymmetric traveling salesman problem with replenishment arcs. European Journal of Operations Research (2000) 123:408-427

16. Vicky M. On the asymmetric traveling salesman problem with replenishment arcs. Department of Mathematics and Statistics, University of Melbourne, PhD thesis (2000) 